\title{
Pedagogical Effectiveness of Classroom Demonstrations Devices
}

\section{Dr. Tom McCormick, Virginia Military Institute}

30 Years of service with the US Army. Retired as COL. 37 Years of services with the Federal Goverment. Retired as a Senior Excutive. Sevred as a sytems enginner. Focued on special operations and counterterrorism. Currently teaching Electrical Enginerring at VMI.

\section{Dr. James C. Squire, Virginia Military Institute}

James Squire is a Professor of Electrical Engineering at the Virginia Military Institute. Dr. Squire received a B.S. from the United States Military Academy and his Ph.D. from the Massachusetts Institute of Technology. He was awarded a Bronze Star in the Army during Desert Storm and was selected as Virginia's Rising Star professor in 2004. He is a licensed Professional Engineer in Massachusetts and Virginia and maintains an active consulting practice.

\section{Prof. Gerald Sullivan P.E., Virginia Military Institute}

Dr. Gerald Sullivan, Associate Professor of Mechanical Engineering at the Virginia Military Institute, received his B.S.M.E. from the University of Vermont and his Ph.D. from Rensselaer Polytechnic Institute. He has held teaching positions at the University of Michigan-Dearborn, and the University of Vermont. Prior to joining the faculty at the Virginia Military Institute in the fall of 2004, he was employed by JMAR Inc. where he was involved in research and development of X-ray lithography systems for the semiconductor industry. His interests include mechanical design, acoustics applications and controls. 


\section{Pedagogical Effectiveness of Classroom Demonstrations Devices}

\section{Abstract}

Classroom demonstrations have been shown to enhance student learning retention by providing greater intellectual stimulation when compared to lecture-only presentations $[4,6,9]$. Yet, comparatively little research has been conducted to determine the presentation type and style that is most effective in pedagogical demonstrations. This investigation builds upon previous work which counterintuitively found that crudely-built demonstrations devices tend to confer greater learning retention than similar professionally built devices [12]. Specifically, we sought to determine how preference for crudely vs. professionally-built demonstrations changed with academic maturity. The current study employed a larger population of students pursuing an undergraduate degree in Science, Technology, Engineering and Math (STEM) in their freshman or sophomore years. The results of the investigation revealed that both year groups experienced greater learning retention when the low-cost, homemade demonstrator was employed when compared to the professionally manufactured demonstrator $(\mathrm{p}=0.04)$. The results also show no significant difference in learning retention between year groups $(\mathrm{p}=.19)$.

Introduction

Pedagogical demonstrations have been proven to effectively enhance student learning and retention. Classroom demonstrators provide a means of intellectual stimulation that can capture student imagination resulting in an improved understanding and a greater retention of lecture material. This research examines two types of pedagogical demonstrations which are functionally equivalent but differ significantly in appearance, price and construction quality. The first consists of a demonstration device which has been homemade. It is simply constructed using components found in any hardware store. The device has been left in its raw form and is crude in appearance. No attempt has been made to make the device aesthetically pleasing. The second type of demonstrator is a professionally manufactured device which has been specifically constructed for classroom use. It has been designed to clearly delineate its individual components and demonstrate their functionality in an aesthetically pleasing manner. This research compares the effectiveness of these devices in order to determine which type of device is most conducive to student learning and retention.

\section{Prior Research}

This investigation builds upon our prior research which showed that "raw" demonstrations were superior to "polished" demonstrations for all measures of learning [12]. This research differentiated raw and polished demonstrations by characteristics listed in Table 1. However, the small sample size necessitated additional study to validate these findings. Additionally, we wished to investigate these findings to determine sensitivity to academic maturity by comparing the results for both freshman and sophomores. 
Table 1: Characteristics differentiating polished and raw demonstrations

\begin{tabular}{|l|l|}
\hline \multicolumn{1}{|c|}{ Polished } & \multicolumn{1}{c|}{ Raw } \\
\hline Learners could buy it & Learners could make it \\
\hline Enclosure tends to hide some of the workings & No enclosure \\
\hline Demonstrates multiple modes of operation & Demonstrates single mode of operation \\
\hline Painted, machine-made appearance & Rough, handmade appearance \\
\hline
\end{tabular}

Further research since the time of our previous investigation has contributed to the body of knowledge concerning classroom demonstrations and their associated impact on student learning and retention. Polizzotto and Tamari showed how simple demonstrations enhanced student performance and improved their ability to visualize, understand and retain biological concepts [11]. Giridharan and Raju found that the use of classroom demonstrations resulted in highly significant gains in academic achievement when compared to lecture-only presentations among engineering students. They also found that in-class demonstrations provoked student interest and were effective for long term memory retention [5]. Cabibihan also showed that using working models as demonstrators for in-class presentations yielded a favorable response from students as well as a greater appreciation for the practical applications of mechatronic concepts [2]. Basheer et al. used statistical analysis to highlight that significant differences existed between students who had and had not been exposed to demonstrations of scientific concepts. They found that chemistry students prefer demonstrations because they are more enjoyable and interesting than lecture-only lessons. Furthermore, the increased interest and enjoyment led to better understanding and greater achievement [1].

Using analysis of covariance at a 0.05 level of significance, Daluba revealed that demonstrations had a significant effect on student achievement when compared to conventional lectures. Specifically, he found that there was a significant difference $(\mathrm{p}<0.05)$ in the mean achievement scores of agricultural science students which favored demonstrations over traditional lecture methods [3]. Manduca et al. focused on improving classroom learning by incorporating demonstrations into the lecture portion of undergraduate science, technology, engineering and mathematics (STEM) education programs. Faculty members are increasingly using more demonstrations and other learning strategies when compared to traditional lecture-only methods. They found a 47\% usage in 2004, a 49\% usage in 2009 and 57\% usage in 2012 and a corresponding decrease in usage of traditional methods. They attribute this change in part to increased awareness of the superiority of demonstration-based methods.

At the 2013 ASEE Southeast Conference, Kunz revealed that his mechanical engineering students often struggle with abstract concepts such as forces, moment and stresses. To combat this he used simple but effective physical demonstrations. All of his demonstrations were constructed from household materials or purchased at the local discount store. He anecdotally concluded that student feedback after viewing the demonstrations was positive and that the demonstrations effectively enabled his students to connect abstract concepts with physical reality [7]. These findings are consistent with the work of Klosky and Schaff who had earlier evaluated the use of demonstrations in mechanical engineering classrooms at West Point [6]. Additionally, Nguyen et al. examined student response to changes in traditional lecture methods by 
engineering instructors in their 2016 ASEE Annual Conference paper. They found that student response was overwhelmingly positive and that instructor concerns about student resistance to change were largely unfounded. They also suggest that additional analysis is needed to determine the salient factors which influence student responses to in-class demonstrations.

While the evidence continues to mount that in-class demonstrations are a highly effective means of improving student learning retention and that demonstrations are increasingly used in a wide array of STEM related undergraduate courses, more investigations are needed concerning the effectiveness of various demonstration designs, functionality and appearance. Our current investigation is therefore focused on obtaining statistically significant results to help guide the STEM education community on the use of the most effective pedagogical demonstration devices.

\section{Demonstration Description}

This investigation compares two types of pedagogical demonstration devices. As previously mentioned, the first is a home-build or "raw" device which is simple and inexpensive to construct while the second demonstrator is a professionally manufactured or "polished" device. Both devices are described in detail below.

The homemade demonstrator is built on a small block of wood and uses L-shaped metal brackets as a stator to hold the hand-wound wire rotor. The stator uses four neodymium magnets placed on top of one another using plastic spacers to create a magnetic field. The current source consists of a 6 Volt/1.5 Amp lead-acid battery. Electrical contact is established via alligator chips attached to the metal brackets.

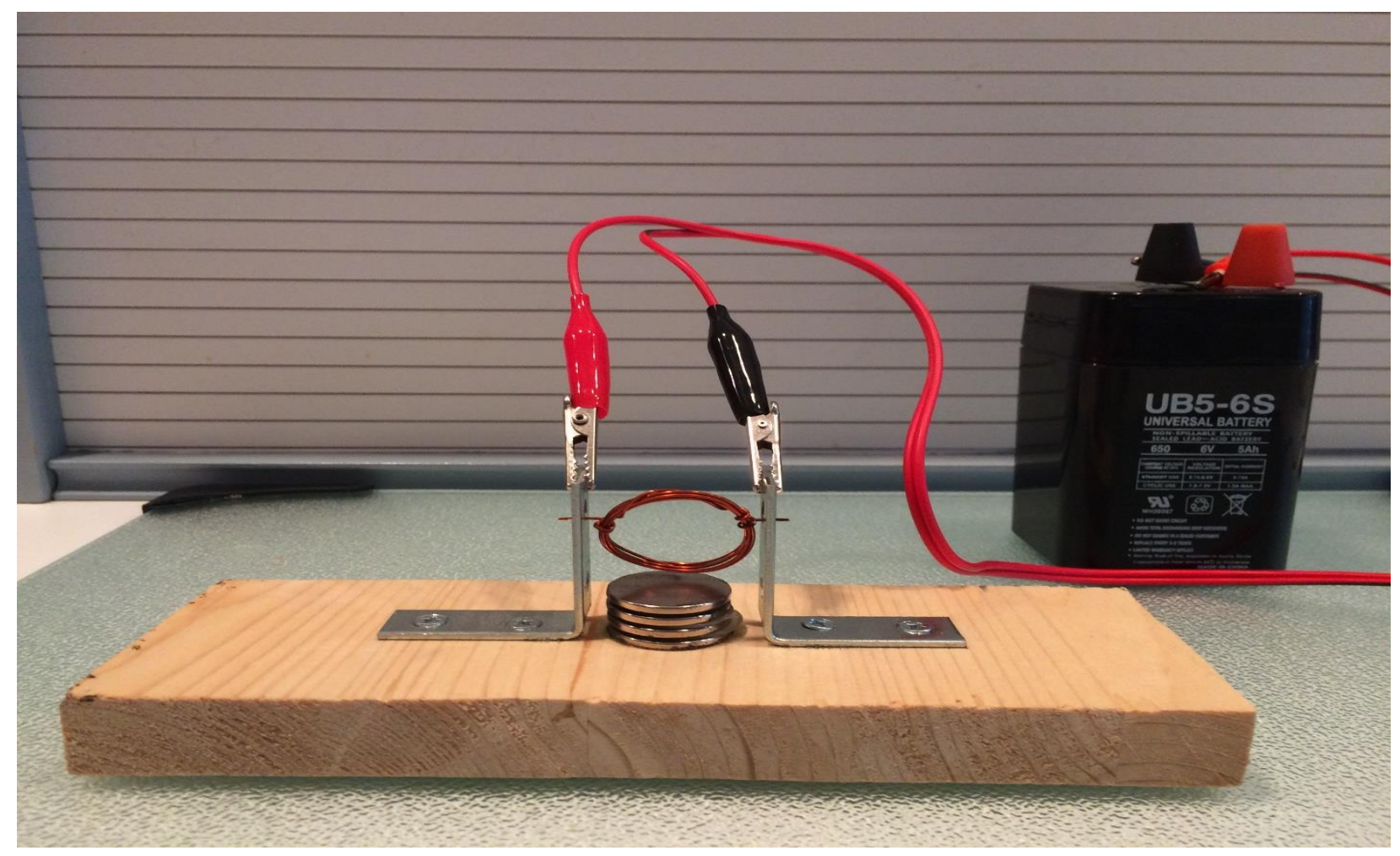

Fig. 1 The "raw" demonstration of an electric motor 
The brackets in turn make intermittent contact with the ends of the copper wire rotor. The electric current flowing through wire in the magnetic field produces a torque which causes the rotor to spin. A commutator function is achieved by sanding the two ends of the copper wire so that approximately 180 degrees of the wire's surface makes electrical contact with the metal bracket during rotation. This ensures that the momentum of the spinning wire can rotate through the reverse torque condition caused by the DC power source. The net result of this construction methodology is a crude or "raw" looking demonstration device as pictured above (Fig. 1).

In sharp contrast to the homemade device is the "polished" demonstrator (Fig. 2). The device consists of color-coded components arranged in an aesthetically pleasing form. Red painted components delineate the positive side of the motor while blue components are of negative polarity. A magnet which is half red and half blue is mounted at the top of the motor and is easily viewable during demonstrations. The magnet sit atop two curved metal brackets which surround the rotor thereby clearly delineating the motor's stator.

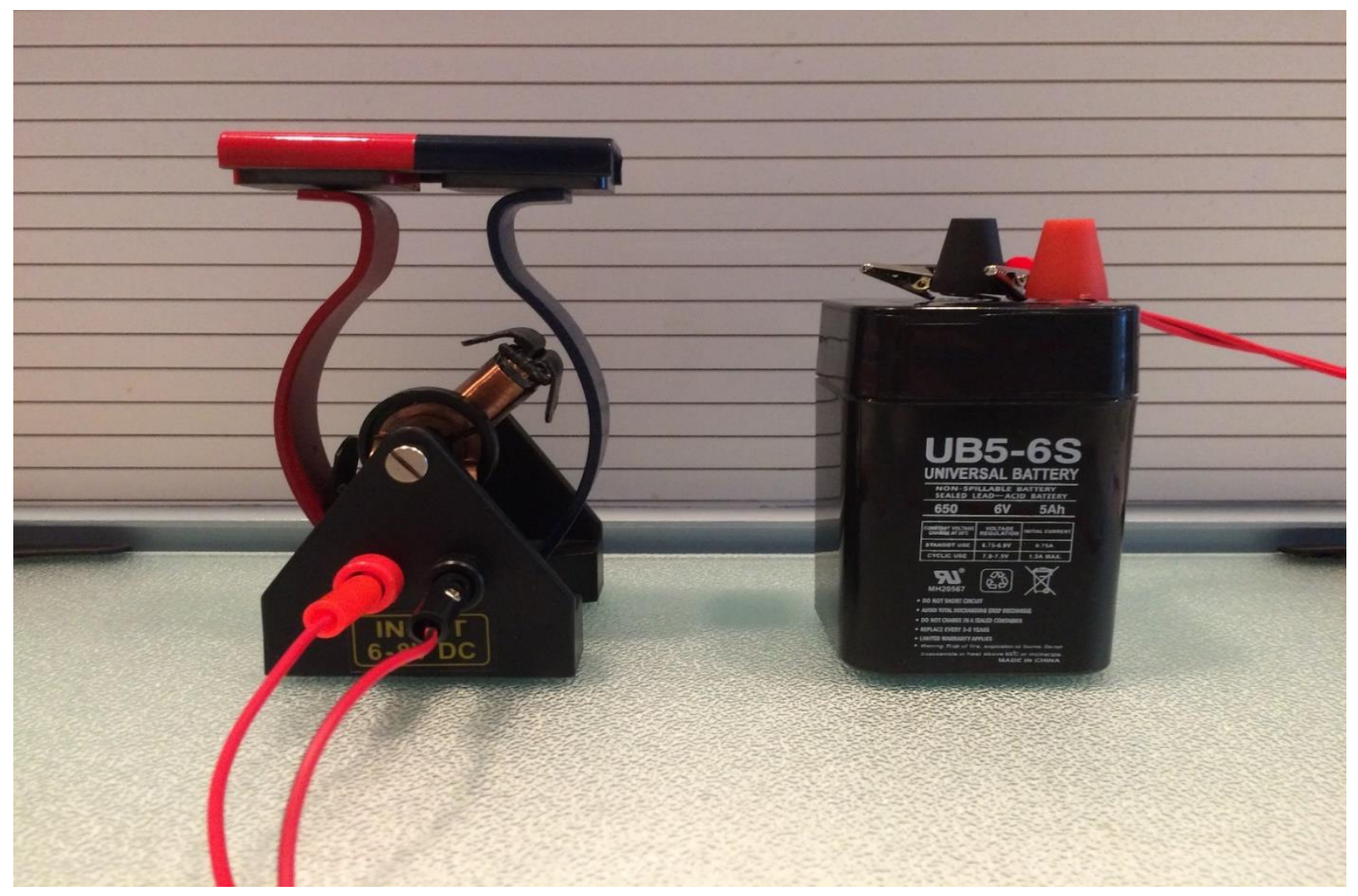

Fig. 2 The "polished" commercially-bought motor demonstration

Electrical connection is made with the battery by the use of two brushes which are visible at the front center of the demonstrator. The circular commutator is also clearly visible and prominently featured to maximize student understanding of how the DC power source enables the rotor to spin continuously. The assembled product is both aesthetically pleasing and successfully packaged in a manner conducive to visualizing the major components while promoting the student's understanding of the functionality of a DC electric motor. 


\section{Demonstration Methodology}

A PowerPoint presentation was used to set the stage for the demonstration and to ensure uniform presentation among different lecturers. The presentation covered the background needed for understanding the demonstration and the learning points for successfully completing the questionnaire which the students filled out immediately following the lecture. The primary teaching points are summarized in condensed form below.

- The lecture began by discussing the industrial revolution and the need for power sources to drive the machines which transformed our modern society.

- Water wheels were replaced by generators and the electric motor, which is essentially a generator operated in reverse, began to drive the machines.

- Wide-scale use of DC electric motors began in the late 1800's.

- These motors take advantage of the properties of electric and magnetic fields.

- Electric current flowing in a wire will produce a circular magnetic field around the wire.

- Magnetic fields produce a force on a current carrying wire. Torque is generated when the windings on each side of the rotor experience forces in opposite directions.

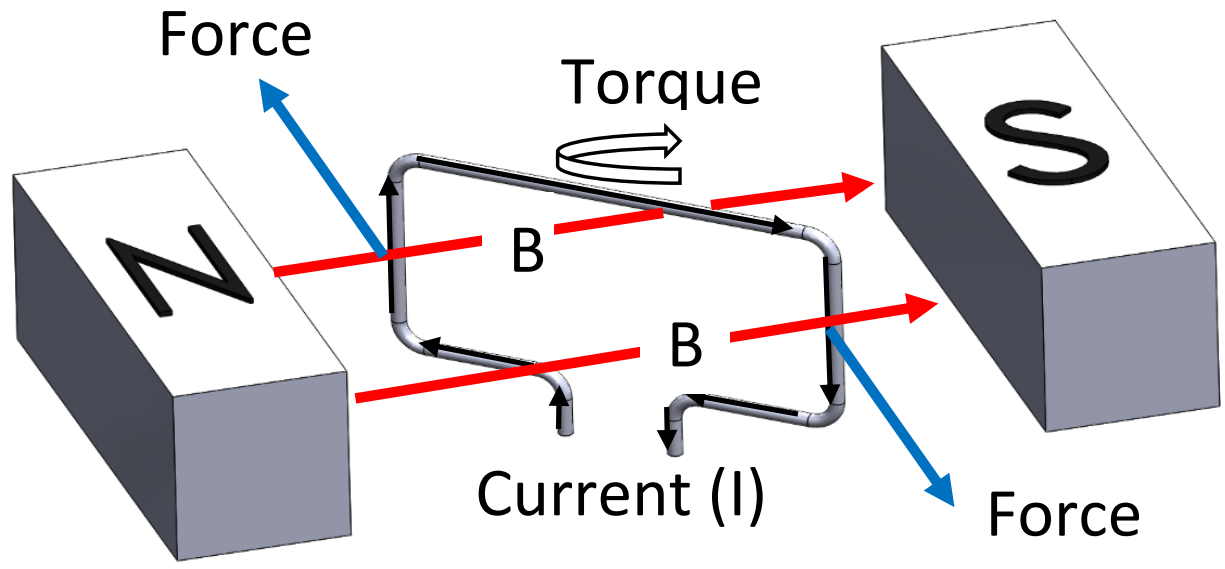

Fig. 3 Schematic operation of an electric motor

- The torque produced by an electric motor is a function of the number of windings, the magnitude of the current, the strength of the magnetic field (B) and the area of the windings.

- A commutator is necessary in a DC motor to change the direction of the current flow every 180 degrees in order to keep the rotor smoothly spinning in one direction.

- The two main components of an electric motor are the rotor and the stator.

- The motor components were pointed out to the students on the demonstration device.

- The battery was connected to the device, allowing students to observe its operation and ask final questions.

- Students were handed a paper copy of the questionnaire and given ten minutes to complete it. 


\section{Questionnaire Method and Description}

Following the standard lecture and the variable demonstration (raw or polished), students were given a standard questionnaire, shown in Appendix I. It consists of three major components. The first component measures the students' subjective enjoyment of the material being presented. It consists of a single question asking students to self-rate how much they enjoyed the material on a Likert scale. The second component measures the students' subjective comprehension of the presentation. This component consists of one question asking students to self-assess how well they thought they learned the material, on a Likert scale, after viewing the demonstration. Finally, the objective comprehension component measures how well the students actually learned the material. This component consists of seven multiple-choice questions drafted by the first author that cover the main teaching points of the presentation. Face validity of these objective questions was established by having the coauthors complete the questions and modify ones they found unclear. Student objective comprehension scores are calculated as the simple ratio of questions correctly answered divided by the total number of questions.

\section{Results}

Data was collected in the months of November and December, 2017 during normal class times at the Virginia Military Institute. A total of 127 students in STEM majors responded to the questionnaire including 63 for the raw demonstration device and 64 for the polished device. The mean subjective enjoyment score for both groups was 0.8 indicating a moderately high level of subjective enjoyment regardless of the type of device used. The mean subjective compression score for the polished device was 0.89 and 0.91 for the raw device. There is no statistical difference between these two numbers and we therefore conclude that both groups of students felt highly confident in their understanding of the material regardless of the type of device used during the demonstration.

A significant difference was noted in the objective comprehension scores between the two groups of students. The objective comprehension score for students taught using the raw device was 0.66 while the polished device results were considerably less at 0.59 . A T-test was used to determine statistical significance which yielded a p-value of 0.04 . We can thus conclude that the raw device is significantly better than the polished device in fostering student learning. The results of all three components of the questionnaire are graphically depicted in Fig. 4 for both the raw and polished demonstration devices. 


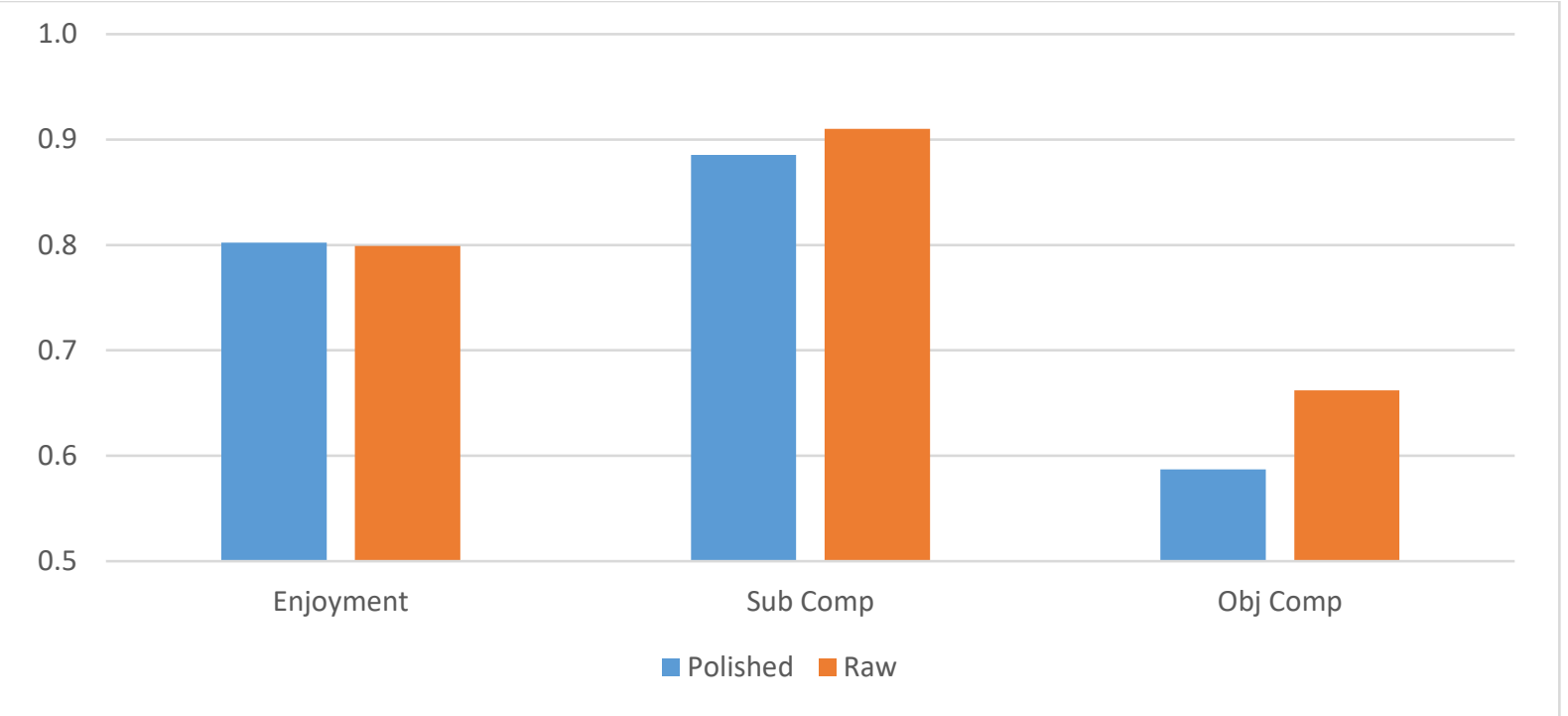

Fig. 4 Results of 127 trials comparing "raw" to "polished" demonstrations

The above findings are consistent with our previous results which showed a statistically significant difference in objective comprehension in favor of the raw demonstration device. The above finding are also consistent with our previous study which concluded that there is no statistically significant difference between demonstration devices for subjective comprehension. However, our previous investigation did show a statistically significant difference in subjective enjoyment which not appear in our current results.

Concerning our current interest in how academic maturity affects objective comprehension, we found no significant difference between freshman and sophomores. This could be due in part to the small sample size of only 17 sophomores for the raw demonstration and 16 for the polished demonstration. The results are graphically portrayed in Fig. 5.

\section{OBJECTIVE COMPREHENSION FOR SOPHOMORES}

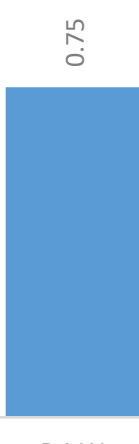

RAW

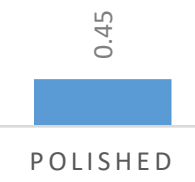

Fig. 5 Comparison of "raw" and "polished" data for sophomores 
Note that that the difference in objective comprehension is even more pronounced for sophomores than the larger data set indicates. Again the small sample size could not establish that this observation met the test for significance but raises some interesting questions to be addressed by future investigations.

\section{Discussion}

Perhaps the most glaring discrepancy between this study and our previously published results can be found in the subjective enjoyment component data. Our previous study showed a significant difference $(\mathrm{p}<.01)$ between raw and polished demonstrations. This difference indicated that student interest in a given lesson is enhanced through the use raw demonstration devices as compared to polished and potentially more expensive devices. Analyzing the previous data in greater detail revealed that a large number of students majoring in non-technical fields were included in this data set but none were included in our current data set. Our previous investigation found that non-technical majors overwhelmingly preferred the raw demonstration device. Perhaps this is because students can relate better to a device that appears to be something they could have made themselves. The rationale behind this preference is therefore an area for further investigation.

Although not a preplanned part of our investigation, it is interesting to compare subjective and objective comprehension scores of the STEM students in our current investigation. The subjective comprehension score for the group exposed to the raw demonstration device was .91 whereas the objective score was 0.66 . This represents a $27 \%$ decrease between student selfassessment and actual achievement. Similarly, the subjective score for the polished demonstration device was 0.89 and the objective score was 0.59 , a $34 \%$ decrease. Both data sets indicate that the student's actual learning is less than their self-evaluation would otherwise indicate (Fig. 6).

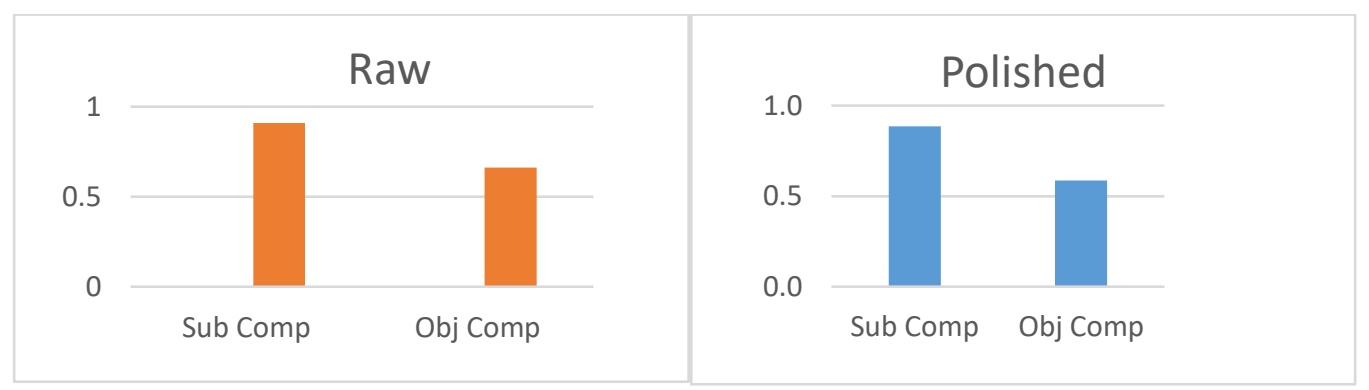

Fig. 6 Comparison of "raw" and "polished" data for subjective and objective comprehension

This side by side comparison of the comprehension results tends to indicate that the student's self-assessed learning is not an accurate representation of their actual learning. This finding has implications for pedagogical investigations where only student self-assessed inputs are used to determine the value of teaching methods and instructional aids. We therefore suggest that some measures of both objective and subject comprehension be used in making these types of assessments. 
Analysis of the objective comprehension scores of the two groups revealed a 7\% lower mean for the group that was exposed to the polished demonstration device. One of the key objective comprehension questions that drove this difference was question number ten in the questionnaire. This question was the most frequently missed question and was missed more often by students who were exposed only to the polished demonstration device. 44 students missed this question after seeing the polished demo and 26 students missed this question after seeing the raw demo. The question was posed as follows:

10) The two main component of an electric motor are

a) Brushes and commutators

b) A commutator and a rotor

c) A rotor and a stator

Note the word "commutator" appears in both incorrect answers, a and b. Since the commutator was prominently visible in the polished demo but not in the raw demo, this may explain why students exposed to the polished demo were more likely to select either a or b. The raw demonstrator has no visible commutator and because of its simplicity students only see the rotor and stator. This may explain why students exposed only to the polished demo were almost twice as likely to incorrectly respond to question number ten.

Question number nine also posed some difficulty of both set of students even though there were only two answers to choose from. Question nine was posed as follows:

9) Direct Current (DC) motors require

a) A commutator

b) No commutator

Student exposed only to the raw demo answered this question incorrectly more often than the group exposed to the polished demo. 19 students answered this question incorrectly after viewing the raw demo compare to only 10 for the polished demo. Again the difference was almost two to one but this time in favor of the polished demo. Again, since there is no visible commutator this may explain why the students viewing the raw demo did not select the correct answer as often as those exposed to the polished device.

During the presentation and prior to activating the demonstration with electric current, some of the students viewing the raw device expressed some doubt as to whether the device would actually work. This may have been because of its rough appearance, simplicity or perhaps the nature of home-built devices. Regardless of the reason, these students tended to pay very close attention to the demonstration whereas the students viewing the professionally-built device expected it to work without question. The element of doubt associated with the raw device may have actually been an unexpected inducement to pay attention and consequently score higher on the objective comprehension portion of the questionnaire.

\section{Conclusion}

This investigation has highlighted once again that the type of demonstration device used in the course of instruction can play an important role in student learning and retention. The 
homemade demonstration device was proven to be significantly better $(\mathrm{p}<0.05)$ than the professional demonstration model based on the mean objective comprehension scores achieved by students in the two groups. Although we attempted to determine if academic maturity was a significance factor in these results, we found no conclusive difference in objective comprehension between the freshman and sophomores in our study groups. We judge that more investigation is needed to make a definitive claim due to the low number of sophomores participating in this investigation. We also found that the subjective comprehension may not be an accurate indicator of student learning. Nevertheless, the use of low-cost, crudely built devices have proven themselves to be more effective than higher priced commercial demonstration devices. 
References

1. Basheer A., Hugerat, M., Kortam, N. and Hofstein, A., "The Effectiveness of Teachers' Use of Demonstrations for Enhancing Students' Understanding of and Attitudes to Learning the Oxidation-Reduction Concept", EURASIA Journal of Mathematics and Technology Education, 13(3), 555-570, 2017.

2. Cabibihan, J.J., "Education of Student Engagement Pedagogies in a Mechatronics Module: A 4-year Multi-Cohort Study", Journal of NUS Teaching Academy, 3(4), 125149, 2013.

3. Daluba, N.E., "Effect of Demonstration Method of Teaching on Students' Achievement in Agricultural Science", World Journal of Education, 3(6), 2013.

4. Felder, R.M., Woods, D.R., Stice, J.E. and Rugarcia, A., "The Future of Engineering Education II. Teaching Methods that Work", Chemical Engineering Education, 34(1) 2639, 2000.

5. Giridharan, K., Raju R., "Impact of Teaching Strategies: Demonstrations and Lecture Strategies and Impact of Teaching Effect on Academic Achievement in Engineering Education”, International Journal of Education Science, 14(3) 174-186, 2016.

6. Klosky, J.L. and Schaaf, R.V.,"Hands-On Demonstrations in Introductory Mechanics", Proceedings ASEE Annual Conference, 2012.

7. Kunz, R., "Simple Demonstrations in Basic Mechanics Courses", Proceedings ASEE Southeast Section Conference, 2013.

8. Manduca, C.A., Iverson, E.R., Luxenberg, M. et al., "Improving Undergraduate STEM Education: The Efficacy of Discipline-Based Professional Development", Science Advances, 3(2), 2017.

9. Morgan, J., Borroso, L.R. and Simpson, N., “Active Demonstrations for Enhanced Learning”, Proceedings ASEE/IEEE Frontiers in Education Conference, 2007

10. Nguyen, K.A., Borrego, M.J., Finelli, C., et al, "Measuring Student Response to Instructional Practices (StRIP) in Traditional and Active Classrooms", Proceedings ASEE Annual Conference, 2016.

11. Polizzotto, K. and Tamari, F., "Using Lecture Demonstrations to Visualize Biological Concepts", Journal of Microbiology and Biology Education, 16(1), 79-81, 2015

12. Sullivan, G.A., Squire, J.C. and Brooke, G.M., "The Effects of Engineering Demonstration Design on Learning and Interest", Proceedings ASEE Annual Conference, 2011. 
Appendix I: Questionnaire

Name Instructor Date

1) What is your major field of study (e.g. ME, English), year (e.g. Fresh, Soph) and age? Field Year Age

2) How did the demonstration of the electric motor affect your interest in the subject matter?

a) The demo made me much more interested in the lecture material; I'm likely to find out more about electric motors on my own time because of it.

b) I found seeing the demo made me much more interested in hearing the talk about electric motors. Still I doubt I'll be Googling to learn more about motors in the near future.

c) The demo was interesting in itself but didn't make me want to learn about electric motors, either in the talk or outside of class.

d) The demo was lame and reinforced my opinion that I just wasted 10 minutes of my time.

3) How did the demonstration help you understand the subject matter?

a) Having a chance to examine the demonstration clarified some things that I would probably not have understood from the lecture alone.

b) Having a chance to examine the demonstration showed me that I correctly understood the material about electric motors taught in class but didn't help me learn anything new.

c) The demo might be cool looking, but it didn't help me understand anything about electric motors

d) I honestly didn't bother to look at it much.

4) What are the similarities/differences between electric motors and generators?

a) They are both used to produce electricity

b) They were both invented well before the industrial revolution

c) An electric motor is essentially a generator operated in reverse

d) They operate on completely different physics principles 
5) Electric current flowing in a wire produces
a) Alternating current in one direction
b) Circular magnetic fields
c) Magnetic fields which are parallel to the current flow
d) No measurable magnetic fields

6) Magnetic fields produce
a) Alternating current in one direction
b) A force on a current carrying wire
c) Circular electric fields
d) Parallel electric fields

7) An electric motor produces torque because
a) Electric current always produces a torque in an open circuit
b) The windings on each side of the rotor experience forces in opposite directions
c) There is no relationship between torque and electric motors

8) Torque is a function of
a) The number of windings only
b) The number of windings and the magnitude of the current
c) The number of windings, the magnitude of the current and the strength of the magnetic field
d) The number of windings, the magnitude of the current, the strength of the magnetic field and the area of the windings

9) Direct Current (DC) motors require
c) A commutator
d) No commutator

10) The two main component of an electric motor are

d) Brushes and commutators

e) A commutator and a rotor

f) A rotor and a stator 